\title{
The response profiles of HSPA12A and TCTP from Mytilus galloprovincialis to pathogen and cadmium challenge
}

\author{
Liping You ${ }^{\mathrm{a}, \mathrm{c}}$, Xuanxuan Ning ${ }^{\mathrm{b}}$, Feng Liu ${ }^{\mathrm{d}}$, Jianmin Zhao ${ }^{\mathrm{a}, *}$, Qing Wang ${ }^{\mathrm{a}}$, Huifeng $\mathrm{Wu}^{\mathrm{a}, * *}$ \\ ${ }^{a}$ Key Laboratory of Coastal Zone Environmental Processes and Ecological Remediation, Yantai Institute of Coastal Zone Research, Chinese Academy of \\ Sciences, Yantai 264003, PR China \\ ${ }^{\mathrm{b}}$ Yantai Oceanic Environmental Monitoring Central Station, State Oceanic Administration, Yantai 264006, PR China \\ ${ }^{\mathrm{c}}$ University of Chinese Academy of Sciences, Beijing 100049, PR China \\ ${ }^{\mathrm{d}}$ China Agriculture University (Yantai), Yantai 264670, PR China
}

\section{A R T I C L E I N F O}

\section{Article history:}

Received 17 August 2012

Received in revised form

5 April 2013

Accepted 22 April 2013

Available online 1 May 2013

\section{Keywords:}

Mytilus galloprovincialis

Heat shock proteins

Gene expression

Vibrio anguillarum

Cadmium

\begin{abstract}
A B S T R A C T
Heat shock $70 \mathrm{kDa}$ protein 12A (HSPA12A) is an atypical member of HSP70 family, and the translationally controlled tumor protein (TCTP) is a novel HSP with chaperone-like activity. They are both involved in protecting organisms against various stressors. In the present study, the cDNAs of HSPA12A and TCTP (called MgHSPA12A and MgTCTP) were identified from Mytilus galloprovincialis by RACE approaches. The full-length cDNA of MgHSPA12A and MgTCTP encoded a peptide of 491 and 171 amino acids, respectively. Real-time PCR was employed to analyze the tissue distribution and temporal expression of these two genes after bacterial challenge and cadmium (Cd) exposure. It was found that the transcripts of MgHSPA12A and MgTCTP were dominantly expressed in gonad and muscle, respectively. The expression level of MgTCTP at $48 \mathrm{~h}$ post Vibrio anguillarum challenge was detected to be significantly up-regulated in hepatopancreas $(P<0.05)$. As concerned to Cd exposure, 2.0-fold increase of MgHSPA12A expression compared to that of the control was observed at $48 \mathrm{~h}$ in $5 \mu \mathrm{g} / \mathrm{L} \mathrm{Cd}^{2+}$-treated group, while the expression levels of MgTCTP were significantly decreased after exposed to both 5 and $50 \mu \mathrm{g} / \mathrm{L} \mathrm{Cd}^{2+}$ for $24 \mathrm{~h}$ and $96 \mathrm{~h}$. These results suggested the potential involvement of MgHSPA12A and MgTCTP in the mediation of the immune responses and environmental stress in mussels.
\end{abstract}

(c) 2013 Elsevier Ltd. All rights reserved.

\section{Introduction}

Heat shock proteins (HSPs) are a family of proteins involved in response to environmental, physical and chemical stresses [1]. According to their molecular masses and functions, HSPs were divided into several families, including HSP100, HSP90, HSP70, HSP60, and the small HSPs (sHSPs) [2]. Heat shock protein 70 (HSP70) and the translationally controlled tumor protein (TCTP) are both highly conserved protein widely existed in all eukaryotic organisms [3,4]. Heat shock $70 \mathrm{kDa}$ protein $12 \mathrm{~A}$ (HSPA12A) is a novel and atypical member of HSP70 family possibly with highly specialized function that might go beyond the HSP chaperone function $[5,6]$. The solution structure of TCTP from fission yeast indicated its similarity to a family of small chaperone proteins [3,7], and a more recent report confirmed that TCTP is a novel HSP with chaperone-like activity [8]. As members of HSPs family, HSPA12A

\footnotetext{
* Corresponding author. Tel.: +86 535 2109170; fax: +86 5352109000. ** Corresponding author. Tel.: +86 535 2109190; fax: +86 5352109000.

E-mail addresses: jmzhao@yic.ac.cn (J. Zhao), hfwu@yic.ac.cn (H. Wu).
}

and TCTP was suggested to possess cytoprotective functions in response to various stressors, especially virus or bacterial pathogen, toxic metals and heat shock [9-12]. Recently, the role of HSPs in the stresses defense system has been well documented in mollusks, such as stimulated-expression of HSP70 in Mytilus galloprovincialis by heat shock and $V$. anguillarum stimuli, the up-regulation of TCTP transcript in Venerupis philippinarum against $V$. anguillarum challenge, the over-expression of HSP70 in Ostrea edulis in response to heat and Cd exposures, etc. [11,13-16].

As ubiquitous, sedentary filter feeders broadly inhabiting in the coastal and estuarine areas, bivalve mollusks are easily infected by various pathogens naturally present in their habitat. For example, Vibrio and other pathogenic bacteria were frequently found in M. galloprovincialis $[17,18]$. Among those pathogens, Vibrio species have been demonstrated to be an important cause of diseases in shellfish [19], which could induce cytotoxicity and the production of reactive oxygen (ROS), etc. [20]. Furthermore, bivalve mollusks are also readily exposed to metallic xenobiotics [21,22]. As an important toxicant xenobiotic, $\mathrm{Cd}$ posed great threat to the marine organisms by generating oxidative stresses after penetrating into cells through calcium channels [15]. 
M. galloprovincialis is an important seafood product and a sentinel species for many countries [23]. In the present study, we aimed to clone the full-length cDNA of HSPA12A and TCTP from $M$. galloprovincialis, and also investigate the temporal expression of these two genes responded to Vibrio anguillarum stimulation and Cd exposure, for better understanding of the defense mechanisms of mussels responded to pathogen and toxic metal stresses.

\section{Materials and methods}

\subsection{M. galloprovincialis and tissues collection}

M. galloprovincialis (shell length $6.5-7.5 \mathrm{~cm}$ ) were collected from a local aquafarm, and maintained at $23{ }^{\circ} \mathrm{C}$ to acclimate for 10 days before commencement of the experiment. The seawater was totally changed every day, and the mussels were fed with unicellular algal species Chrysophyta and Tetraselmis chui daily. The tissues, including hemocytes, hepatopancreas, muscle, gonad, gills, and mantle, were dissected from six individuals as parallel samples to investigate the tissue-specific expression of MgHSPA12A and MgTCTP transcripts. These tissues were subjected to total RNA extraction by using TRIzol reagent (Invitrogen) immediately.

\subsection{Bacterial challenge and toxic metal exposure experiments}

A total of 300 mussels were employed in $V$. anguillarum chanllenge and Cd exposure experiments, and they were classified into four groups. Mussels of the control group were cultured in the filtered seawater. For the bacterial challenge, mussels were intramuscularly injected with $10^{6} \mathrm{CFU}$ live $V$. anguillarum according to the method described by Cellura et al. It has been demonstrated that mechanical operations, such as handling, filing and needle insertion, exerted little effects on the variations of HSPs gene expressions [13]. During the toxic metal exposure, mussels in the other two groups were treated with environmentally relevant concentrations of $\mathrm{Cd}$ (prepared from $\mathrm{CdCl}_{2}$ ) at 5 and $50 \mu \mathrm{g} / \mathrm{L}$, respectively. The concentrations of $\mathrm{Cd}$ have been previously reported in some heavily polluted sites of the Bohai Sea [24]. The $\mathrm{CdCl}_{2}$ solutions were added to the seawater when the seawater was refilled every day. After bacterial challenge and Cd exposure, the hepatopancreas of six individuals were dissected from the control (at $0 \mathrm{~h}$, as the naive specimens) and treated groups at each time intervals ( $24 \mathrm{~h}, 48 \mathrm{~h}$ and $96 \mathrm{~h}$ ), respectively, and subjected to total RNA extraction immediately.

\subsection{Cloning the full-length cDNA of MgHSPA12A and MgTCTP}

Partial cDNA sequences of MgHSPA12A and MgTCTP were obtained from the subtractive cDNA library constructed from the digestive gland of M. galloprovincialis. Nested PCR was employed to clone the full-length cDNAs of MgHSPA12A and MgTCTP. And the forward and reverse primers were listed in Table 1. The PCR amplification and sequencing were performed according to described previously [25].

\subsection{Sequence analysis}

The cDNA and deduced amino sequences were analyzed by using the BLAST algorithm at National Centre for Biotechnology Information (http://www.ncbi.nlm.nih.gov/blast). Multiple alignments were performed using the ClustalW Multiple Alignment Program (http://www.ebi.ac.uk/clustalw/), and the signal peptide was predicted by using the SignalP 3.0 software. Two phylogenetic trees were constructed according to the amino acid sequences of HSPA12A and TCTP by using neighbor-joining method embedded in
Table 1

PCR primers used in this study.

\begin{tabular}{|c|c|c|}
\hline Primers & Sequence $\left(5^{\prime}-3^{\prime}\right)$ & Primer information \\
\hline P1 (forward) & TTCTTTGTGATTACCGTTCC & $\begin{array}{l}\text { MgHSPA12A } \\
\text { specific primer }\end{array}$ \\
\hline P2 (forward) & ATGATGTTAGCCTATGAGCC & $\begin{array}{l}\text { MgHSPA12A } \\
\text { specific primer }\end{array}$ \\
\hline P3 (reverse) & CGGCTCATAGGCTAACATC & $\begin{array}{l}\text { MgHSPA12A } \\
\text { specific primer }\end{array}$ \\
\hline P4 (reverse) & AGGAACGGTAATCACAAAG & $\begin{array}{l}\text { MgHSPA12A } \\
\text { specific primer }\end{array}$ \\
\hline P5 (forward) & ATGGATTACAGGGAAGACGG & $\begin{array}{l}\text { MgTCTP } \\
\text { specific primer }\end{array}$ \\
\hline P6 (forward) & AACTGGTGCTATGGATGCTG & $\begin{array}{l}\text { MgTCTP } \\
\text { specific primer }\end{array}$ \\
\hline P7 (reverse) & CATCCATAGCACCAGTTCG & $\begin{array}{l}\text { MgTCTP } \\
\text { specific primer }\end{array}$ \\
\hline P8 (reverse) & GTGTTACTCCGTCTTCCCT & $\begin{array}{l}\text { MgTCTP } \\
\text { specific primer }\end{array}$ \\
\hline P9 (forward) & GTTAGCCTATGAGCCAGAAGCG & $\begin{array}{l}\text { MgHSPA12A } \\
\text { specific primer } \\
\text { for RT-PCR }\end{array}$ \\
\hline P10 (reverse) & CAGCCGTACCACCACCAAGAT & $\begin{array}{l}\text { MgHSPA12A } \\
\text { specific primer } \\
\text { for RT-PCR }\end{array}$ \\
\hline P11 (forward) & CATAATGCAATCACTACGCTCTG & $\begin{array}{l}\text { MgTCTP specific } \\
\text { primer for RT-PCR }\end{array}$ \\
\hline P12 (reverse) & ATCGTCGTCGTAACAATGTCAAG & $\begin{array}{l}\text { MgTCTP specific } \\
\text { primer for RT-PCR }\end{array}$ \\
\hline Actin-F (forward) & GCTATCCAGGCCGTACTCT & $\beta$-actin primer \\
\hline Actin-R (reverse) & GCGGTGGTTGTGAATGAG & $\beta$-actin primer \\
\hline
\end{tabular}

Mega4.1 program. To test the relative support for the phylogeny analysis, bootstrap trials were replicated 1000 times.

\subsection{Quantification analysis of $m R N A$ expression}

The temporal expression of MgHSPA12A and MgTCTP to $V$. anguillarum infection and Cd exposure was measured in Applied Biosystem 7500 fast Real-time PCR System. Two pairs of genespecific primers (Table 1), were designed to amplify PCR products of $125 \mathrm{bp}$ and $164 \mathrm{bp}$ respectively. The products were sequenced to verify the PCR specificity. The housekeeping gene $\beta$-actin (primers information listed in Table 1) was used as the internal control to verify the successful transcription and to calibrate the cDNA template for corresponding mussel samples [26,27]. In a 96-well plate, each sample was run in triplicate along with the internal control. The hepatopancreas RNA extraction, cDNA synthesis, reaction component, thermal profile and the data analysis were conducted as previously described [28]. Dissociation curve analysis of amplification products was performed at the end of each PCR reaction to confirm that only one PCR product was amplified and detected. The $2^{-\triangle \triangle C T}$ method was used to analysis the relative expression level of the two genes [29].

\subsection{Statistical analysis}

One-way analysis of variance (one-way ANOVA) was performed on all data using SPSS 16.0 statistical software, and $p$ values less than 0.05 were considered statistically significant. All data were given as means $\pm \operatorname{SE}(n=6)$.

\section{Results}

\section{1. cDNA cloning and sequence analysis of MgHSPA12A and MgTCTP}

Two nucleotide sequences of 2039 and $950 \mathrm{bp}$ representing the complete cDNA sequence of MgHSPA12A and MgTCTP were 


\section{A}

1 TTTCTAGTTTAGTGCTAACAAGGAAGTTAATTAATAAGAAGGTCACAATGAAAACATCAG 61 CTTTAAATTTGCAATTGAAGTTTTTATGACACAAAATTGACCATGGACTAAATTGTGTGT

1

$\begin{array}{llllllllllll}M & \mathrm{E} & \mathrm{A} & \mathrm{S} & \mathrm{R} & \mathrm{R} & \mathrm{T}\end{array}$

121 AGACTGCAGGTTAGCGGCGTTAAGAGGTGTTTAAAACAAAAATGGAAGCATCAAGGAGAA

$\begin{array}{lllllllllllllllllllll}8 & \text { S } & \text { E } & \text { C } & R & G & S & R & \text { L } & \text { V } & \text { V } & \text { A } & \text { A } & \text { I } & \text { D } & \text { L } & G & \text { T } & \text { T } & F & S\end{array}$ 181 CATCAGAATGCAGAGGAAGTCGGTTAGTTGTTGCTGCTATAGACTTGGGAACAACTTTCT $\begin{array}{lllllllllllllllllllll}28 & G & \text { Y } & \text { A } & \text { F } & \text { S } & \text { F } & \text { K } & \text { S } & \text { D } & \text { F } & \text { E } & \text { S } & \text { N } & \text { P } & \text { L } & \text { K } & \text { I } & \text { S } & \text { T } & \text { N }\end{array}$ 241 CAGGATATGCATTTTCCTTCAAATCTGATTTTGAGTCCAACCCACTCAAAATTAGCACAA $\begin{array}{lllllllllllllllllllll}48 & \text { D } & \text { W } & \text { S } & \text { A } & \text { E } & G & \text { V } & \text { H } & \text { S } & \text { S } & \text { E } & \text { M } & \text { K } & \text { A } & \text { P } & \text { S } & \text { I } & \text { L } & \text { L } & \text { L }\end{array}$ 301 ATGATTGGTCAGCAGAGGGAGTTCACAGTTCAGAGATGAAAGCGCCATCTATATTGTTGC $\begin{array}{lllllllllllllllllllll}68 & \text { N } & \text { P } & \text { D } & \text { Q } & \text { S } & \text { F } & \text { N } & \text { C } & \text { F } & \text { G } & \text { Y } & \text { R } & \text { A } & \text { Q } & \text { H } & \text { T } & \text { Y } & \text { S } & \text { D } & \text { L }\end{array}$ 361 TAAATCCTGACCAATCATTTAACTGCTTCGGATACCGAGCACAACACACTTACTCAGACT $\begin{array}{lllllllllllllllllllll}88 & \text { L } & \text { D } & \text { D } & \text { D } & \text { P } & \text { D } & \text { K } & \text { A } & \text { A } & \text { K } & \text { Y } & \text { Y } & \text { F } & \text { V } & \text { K } & \text { N } & \text { F } & \text { K } & \text { M } & \text { Q }\end{array}$ 421 TGTTGGACGATGACCCAGATAAAGCTGCCAAATATTATTTTGTTAAGAACTTCAAAATGC $\begin{array}{lllllllllllllllllllll}108 & \text { L } & H & \text { N } & \text { R } & \text { E } & \text { I } & \text { S } & \text { L } & G & \text { M } & \text { M } & \text { I } & \text { L } & \text { D } & \text { V } & \text { T } & \text { G } & \text { K } & \text { E } & \text { L }\end{array}$ 481 AGCTACACAATAGGGAAATTTCCTTAGGGATGATGATTCTAGATGTAACAGGAAAAGAAT

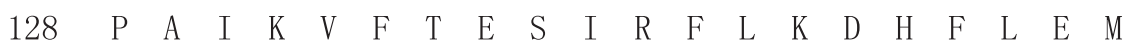
541 TACCTGCTATCAAAGTATTTACTGAATCAATAAGGTTTCTCAAAGACCATTTTCTTGAGA $\begin{array}{lllllllllllllllllllll}148 & \mathrm{~F} & \mathrm{~T} & \mathrm{D} & \mathrm{K} & \mathrm{K} & \mathrm{L} & \mathrm{G} & \mathrm{F} & \mathrm{S} & \mathrm{I} & \mathrm{N} & \mathrm{D} & \mathrm{V} & \mathrm{F} & \mathrm{F} & \mathrm{V} & \mathrm{I} & \mathrm{T} & \mathrm{V} & \mathrm{P}\end{array}$ 601 TGTTTACCGACAAAAAGTTAGGATTCTCTATCAATGATGTGTTCTTTGTGATTACCGTTC $\begin{array}{lllllllllllllllllllll}168 & \text { A } & \text { I } & \text { W } & \text { S } & \text { D } & \text { A } & \text { A } & \text { K } & \text { Q } & \text { F } & \text { M } & \text { R } & \text { V } & \text { A } & \text { S } & \text { E } & K & \text { A } & G & \text { I }\end{array}$ 661 CTGCCATTTGGAGCGACGCTGCAAAACAGTTCATGAGGGTAGCCTCTGAGAAGGCAGGTA $\begin{array}{lllllllllllllllllllll}188 & \text { K } & \text { S } & \text { S } & \text { Q } & \text { M } & \text { M } & \text { L } & \text { A } & \text { Y } & \text { E } & \text { P } & \text { E } & \text { A } & \text { A } & \text { A } & \text { L } & \text { F } & \text { C } & \text { S } & \text { L }\end{array}$ 721 TAAAAAGTTCCCAGATGATGTTAGCCTATGAGCCAGAAGCGGCAGCCTTGTTCTGTAGTC $\begin{array}{lllllllllllllllllllll}208 & \text { L } & \text { P } & \text { E } & \text { D } & \text { Q } & \text { G } & \text { I } & \text { A } & \text { K } & \text { Y } & \text { F } & \text { Q } & \text { E } & \text { R } & \text { R } & \text { R } & \text { L } & \text { M } & \text { I } & \text { V }\end{array}$ 781 TGTTACCCGAGGATCAAGGAATAGCTAAATATTTCCAGGAACGACGAAGACTTATGATAG

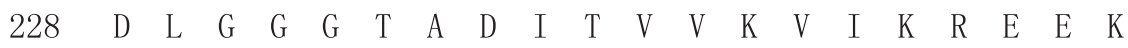
841 TAGATCTTGGTGGTGGTACGGCTGATATAACGGTTGTCAAAGTAATAAAACGTGAGGAGA $\begin{array}{lllllllllllllllllllll}248 & \text { L } & \text { T } & \text { Y } & \text { I } & \text { E } & \text { H } & \text { V } & \text { Y } & \text { R } & \text { V } & \text { T } & \text { G } & \text { G } & \text { A } & \text { W } & \text { G } & \text { G } & \text { N } & \text { Q } & \text { V }\end{array}$ 901 AACTGACCTATATAGAGCACGTGTACAGAGTCACAGGAGGGGCATGGGGCGGTAACCAAG

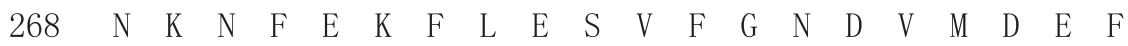
961 TCAATAAGAATTTTGAAAAATTCTTAGAATCGGTATTCGGTAATGACGTCATGGACGAGT

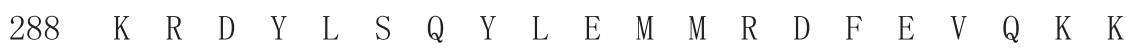
1021 TTAAACGAGATTATCTTTCCCAATATTTGGAGATGATGAGAGATTTTGAAGTGCAAAAGA $\begin{array}{lllllllllllllllllllll}308 & \mathrm{~T} & \mathrm{~L} & \mathrm{~S} & \mathrm{~T} & \mathrm{~S} & G & \mathrm{~T} & \mathrm{~K} & \mathrm{~T} & \mathrm{G} & \mathrm{V} & \mathrm{G} & \mathrm{I} & \mathrm{R} & \mathrm{F} & \mathrm{G} & \mathrm{G} & \mathrm{D} & \mathrm{L} & \mathrm{R}\end{array}$ 1081 AAACACTTTCAACTTCCGGTACGAAAACGGGTGTCGGAATAAGATTTGGAGGAGACCTTC $\begin{array}{lllllllllllllllllllll}328 & \text { H } & \text { I } & \text { F } & K & \text { S } & K & R & G & K & D & \text { I } & K & D & \text { E } & \text { L } & \text { Q } & \text { D } & K & \text { L } & K\end{array}$ 1141 GTCATATTTTTAAAAGTAAGCGAGGAAAAGATATTAAAGATGAATTACAAGACAAACTTA $\begin{array}{lllllllllllllllllllll}348 & G & M & V & R & I & T & G & D & K & \text { L } & R & F & D & \text { L } & \text { A } & \text { I } & \text { F } & \text { K } & \text { R } & \text { F }\end{array}$ 1201 AAGGTATGGTTAGAATTACAGGAGACAAATTGCGATTTGACCTAGCAATCTTCAAAAGAT $\begin{array}{lllllllllllllllllllll}368 & \text { F } & \text { Q } & \text { D } & \text { C } & \text { V } & \text { K } & \text { E } & \text { I } & \text { V } & \text { N } & \text { H } & \text { I } & \text { N } & \text { E } & \text { S } & \text { F } & \text { L } & \text { K } & \text { E } & \text { D }\end{array}$ 1261 TTTTCCAAGACTGTGTAAAAGAGATTGTCAATCACATAAATGAAAGCTTTTTAAAAGAGG $\begin{array}{lllllllllllllllllllll}388 & \text { V } & \text { L } & G & \text { R } & \text { L } & \text { P } & \text { M } & \text { I } & \text { L } & \text { V } & G & G & F & \text { S } & \text { D } & \text { A } & \text { P } & \text { V } & \text { I } & R\end{array}$ 1321 ATGTGCTTGGCCGTCTGCCAATGATACTAGTTGGTGGATTTTCTGATGCTCCTGTAATCC

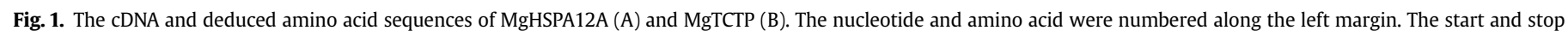
codon were in bold. 
$\begin{array}{lllllllllllllllllllll}408 & \text { E } & \text { A } & \text { I } & \text { V } & \text { D } & \text { A } & \text { F } & \text { P } & \text { V } & \text { I } & \text { K } & \text { D } & \text { V } & \text { V } & \text { S } & \text { P } & \text { V } & \text { A } & \text { T } & \text { S }\end{array}$ 1381 GGGAAGCAATTGTCGATGCTTTTCCCGTTATAAAAGATGTTGTGTCTCCCGTGGCGACTT $\begin{array}{lllllllllllllllllllll}428 & \text { L } & \text { A } & \text { V } & \text { L } & \text { K } & G & \text { S } & \text { V } & \text { I } & \text { F } & G & \text { H } & \text { E } & \text { P } & \text { A } & \text { I } & \text { V } & \text { T } & G & \text { R }\end{array}$ 1441 CATTGGCCGTTTTAAAAGGATCAGTTATTTTTGGTCACGAACCAGCAATAGTTACTGGCC $\begin{array}{lllllllllllllllllllll}448 & \mathrm{~V} & \mathrm{C} & \mathrm{R} & \mathrm{E} & \mathrm{T} & \mathrm{L} & \mathrm{G} & \mathrm{L} & \mathrm{T} & \mathrm{L} & \mathrm{R} & \mathrm{R} & \mathrm{P} & \mathrm{Y} & \mathrm{V} & \mathrm{P} & \mathrm{E} & \mathrm{G} & \mathrm{Q} & \mathrm{T}\end{array}$ 1501 GAGTCTGTCGCGAAACGCTAGGCCTCACACTCCGTCGGCCATATGTGCCGGAAGGTCAAA $\begin{array}{lllllllllllllllllllll}468 & \text { E } & \text { K } & \text { K } & \text { T } & \text { F } & \text { K } & \text { I } & \text { D } & G & \text { Q } & \text { L } & \text { R } & \text { A } & \text { D } & \text { K } & \text { S } & \text { F } & \text { Q } & \text { K } & \text { M }\end{array}$ 1561 CTGAAAAGAAAACGTTTAAAATTGATGGACAACTTCGAGCAGATAAAAGTTTTCAAAAAA

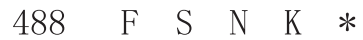

1621 TGTTTTCCAATAAATGAAATCATTAAACTTGGACAAACCCGGTACAGTGCCAATACAGAT 1681 ATTTCATACAAAAAAAACGTTACGAAAACATCCGAAGATTATCGAGGTGTACGCTTCACA 1741 AGACGAGATTCCTGATAATATCACGGCCGCTGGTTTTTCCCTTAGAGGAAAAATCCCTGT 1801 TATTCCCCCAAGGGGAAAAGGGCCGGAAAAGGGGGAGGGTTTTTTTGTTTGGGAAACGGG 1861 GGGAACGGAAATTTTGGTCGGGTCCGGAGCCAACCATCCCGCCCAGATTATGAAGGAGGG 1921 AAAAGTTTTTATGGGATTAATTTTTTTGGAAAAAAGTTTTTTTTCCAAAAATTTGAAAAT 1981 TAAAAAGTTTTTTTTTGGAAAAAAAAAAAAAAAAAAAAAAAAAAAAAAAAAAAAAAAAA

B

1 TTCTGACAGCCGGTGTCGCAACACTTCTGTTTATAACAGAAAATAATCTACCAACATCAG $\begin{array}{llllllllllllllllllll}1 & \text { M } & \text { I } & \text { I } & \text { Y } & R & C & K & \text { I } & \text { S } & G & \text { D } & \text { E } & \text { L } & \text { F } & \text { S } & \text { D } & \text { A } & \text { F } & \text { E }\end{array}$ 61 CCAAAATGATTATTTACAGGTGTAAAATATCAGGAGATGAATTGTTCAGTGATGCTTTTG $\begin{array}{lllllllllllllllllllll}20 & \text { P } & \text { M } & \text { I } & \text { V } & \text { K } & \text { E } & \text { D } & \text { F } & \text { F } & \text { Y } & \text { E } & \text { I } & \text { E } & \text { G } & \text { K } & \text { N } & \text { I } & \text { S } & \text { V } & \text { S }\end{array}$ 121 AACCAATGATTGTGAAAGAAGACTTCTTCTATGAAATTGAGGGAAAGAATATTTCTGTCA $\begin{array}{lllllllllllllllllllll}40 & \mathrm{~N} & \mathrm{~K} & \mathrm{I} & \mathrm{D} & \mathrm{E} & \mathrm{S} & \mathrm{A} & \mathrm{I} & \mathrm{G} & \mathrm{A} & \mathrm{N} & \mathrm{A} & \mathrm{S} & \mathrm{A} & \mathrm{E} & \mathrm{E} & \mathrm{A} & \mathrm{A} & \mathrm{E} & \mathrm{G}\end{array}$ 181 GCAATAAAATTGATGAATCTGCAATTGGAGCCAACGCTTCAGCAGAGGAGGCAGCAGAAG $\begin{array}{lllllllllllllllllllll}60 & \text { Q } & \text { E } & \text { D } & \text { L } & \text { V } & \text { E } & \text { T } & \text { K } & \text { I } & \text { N } & \text { V } & \text { I } & \text { Y } & \text { S } & \text { H } & \text { K } & \text { L } & \text { Q } & \text { E } & \text { T }\end{array}$ 241 GCCAAGAAGACTTAGTAGAAACCAAAATTAATGTAATTTACAGTCACAAACTTCAAGAAA

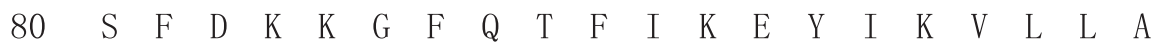
301 CCAGTTTTGACAAGAAAGGTTTCCAGACATTTATCAAAGAATATATTAAAGTATTATTAG

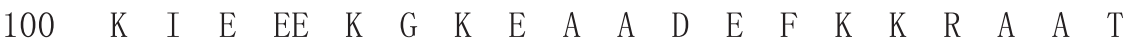
361 CTAAAATTGAAGAAGAAAAAGGCAAAGAAGCTGCAGATGAATTCAAAAAGCGAGCAGCCA $\begin{array}{lllllllllllllllllllll}120 & G & \text { V } & \text { K } & \text { K } & \text { V } & \text { L } & \text { E } & \text { N } & \text { F } & \text { K } & \text { N } & \text { W } & \text { Q } & \text { F } & \text { F } & \text { Q } & \text { G } & \text { E } & \text { N } & \text { M }\end{array}$ 421 CAGGTGTAAAAAAAGTTTTAGAAAACTTCAAAAATTGGCAATTTTTCCAAGGAGAAAACA $\begin{array}{lllllllllllllllllllll}140 & \text { A } & D & G & G & M & \text { I } & \text { V } & \text { L } & \text { M } & \text { D } & \text { Y } & \text { R } & \text { E } & \text { D } & \text { G } & \text { V } & \text { T } & \text { P } & \text { Y } & \text { F }\end{array}$ 481 TGGCTGATGGAGGCATGATAGTATTAATGGATTACAGGGAAGACGGAGTAACACCTTATT $\begin{array}{lllllllllllllll}160 & \text { W } & \text { F } & \text { V } & \text { K } & \text { D } & \text { G } & \text { I } & \text { I } & \text { A } & \text { E } & \text { K } & \text { Y } & *\end{array}$

541 TCTGGTTTGTTAAAGATGGAATTATTGCAGAAAAATATTGAACCTCAAAACTAAGAACTG 601 TAGTTGATATCGGCGAACTGGTGCTATGGATGCTGAACATCTCTCCTGACAATGATAGAA 661 CAGCAGCAAATAGCACGTCTTATTTAACATTTTCATTGTGAAAATCTATACATCCTATAT 721 CATAATGCAATCACTACGCTCTGCTTCAAAGAACCGTCATATTTAAAATTTTTAACCCAA 781 CTATACTATGTATAATTATGGGAACTGTGTATAATTGTGGCATTGATTACAGCTGGTTTA 841 GACCGGGCATTCTAAATATTTCTTGACATTGTTACGACGACGATGATTACATTACCTATT 901 CTATGTCAAATAAAACATGCTATTTATTATAAAAAAAAAAAAAAAAAAAA 

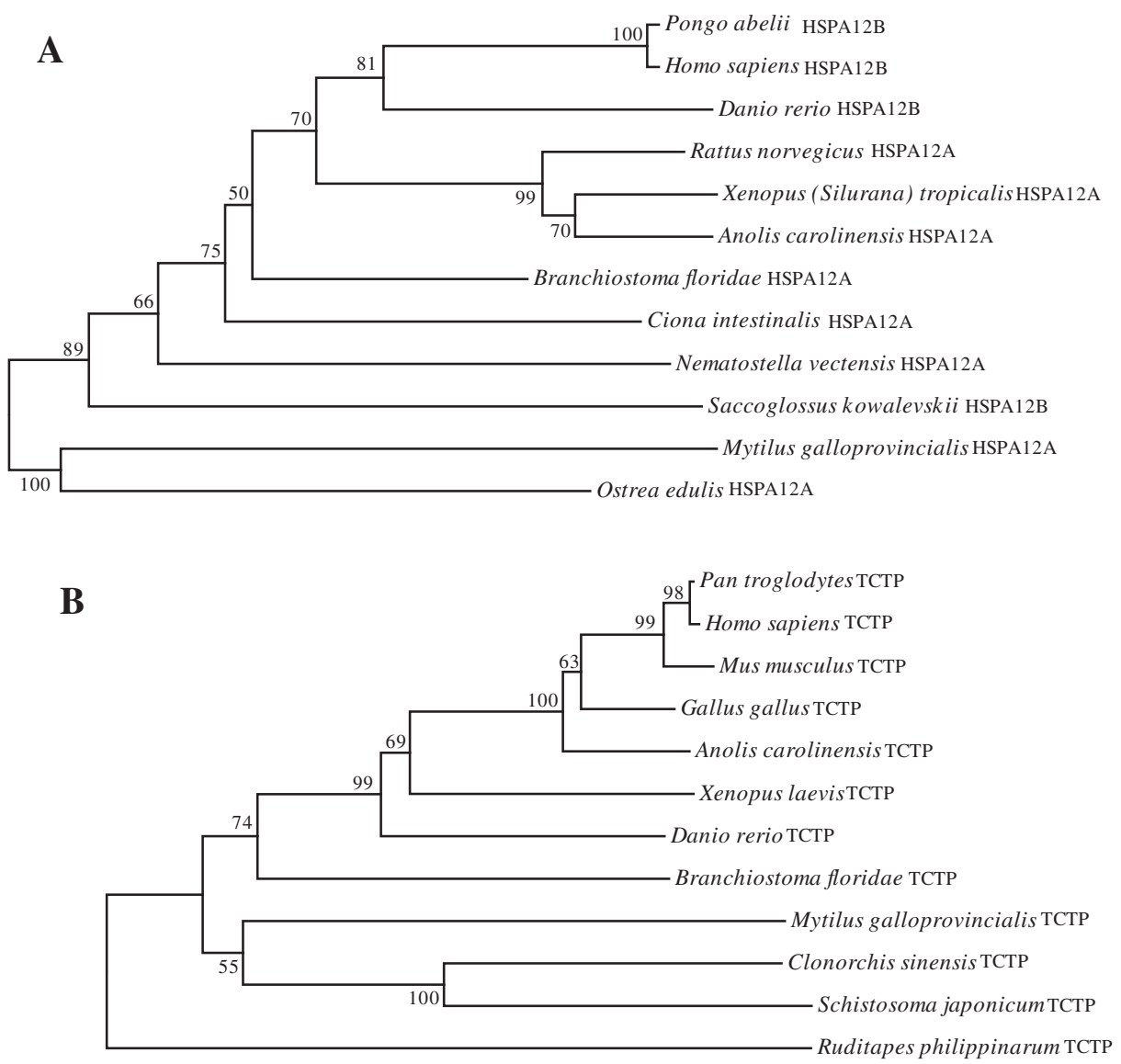

$\longmapsto$

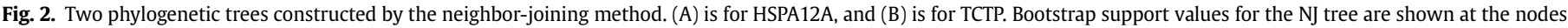

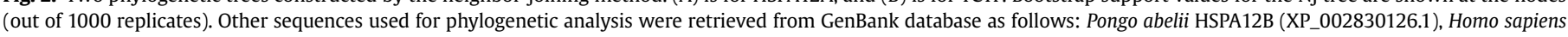

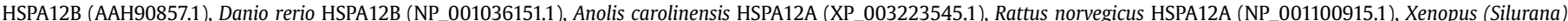

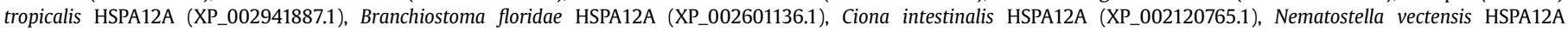

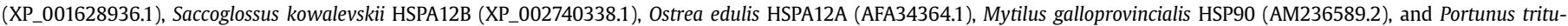

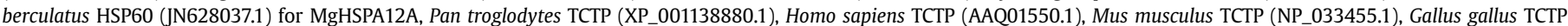

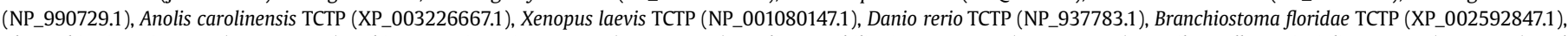

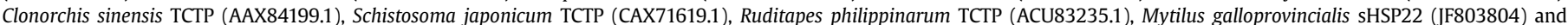
Mytilus galloprovincialis sHSP24.1 (JF803805) for MgTCTP.

obtained by RACE approach, respectively. The deduced amino acid sequences of MgHSPA12A (GenBank accession no. JN232381) and MgTCTP (GenBank accession no. JN232382) were shown with the corresponding nucleotide acid sequence in Fig. 1. The open reading frames of MgHSPA12A and MgTCTP were of 1473 and 516 bp, encoding 491 and 171 amino acids, respectively. No signal peptide was identified in both MgHSPA12A and MgTCTP by SignalP analysis, indicating that the deduced proteins should be cytosolic HSPs.

\subsection{Multiple sequences alignment and phylogenetic analysis}

Multiple sequence alignment of MgHSPA12A with counterparts from other organisms revealed the typical HSP70 family signatures [IV]-D-L-G-T-[ST]- $x$-[SC] and [LIVMF]-[LIVMFY]-[DN]-[LIVMFS]-G[GSH]-[GS]-[AST]- $x(3)-[S T]-[$ LIVM]-[LIVMFC] in the deduced amino acid sequence of MgHSPA12A at 20aa-27aa (IDLGTTFS) and 226aa-239aa (IVDLGGGTADITVV) (Fig. S1A), respectively. Blast analysis revealed that MgTCTP shared high identities with other registered TCTPs as well (Fig. S1B). Two potential protein kinase $C$ phosphorylation sites [ST]-X-[RK], corresponding to the amino acid positions at 39aa-41aa (SNK) and 72aa-74aa (SHK), and one casein kinase phosphorylation site at 9aa-12aa (SGDE) were identified in MgTCTP through Prosite scan. Additionally, GANASA (48aa-53aa) in MgTCTP was predicted to be an N-myristoylation site, which was consensus to its typical sequence G-\{EDRKHPFYW\}-x(2)-[STAGC $\mathrm{N}]-\{\mathrm{P}\}$. One of the characteristic domains of the TCTP family [IFAE][GA]-[GAS]-N-[PAK]-S-[GTA]-E-[GDEV]- [PAGEQV]-[DEQGAV] of TC TP-1 was also found at 47aa-57aa (IGANASAEEAA).

Based on the protein sequences aligned in the multiple sequences, two phylogenetic trees were constructed by the neighborjoining method, respectively. As revealed in Fig. 2A, MgHSPA12A formed the invertebrate group with HSPA12A counterparts from O. edulis, Saccoglossus kowalevskii, Nematostella vectensis, and Ciona intestinalis, and especially shared a common branch with 0 . edulis. Concerning to MgTCTP (Fig. 2B), it was firstly clustered into the invertebrate group with TCTP from Ruditapes philippinarum, Clonorchis sinensis, and Schistosoma japonicum, etc, and then formed a sister group to the vertebrate cluster. The relationships displayed in the phylogenic trees were in good agreement with traditional taxonomy. 

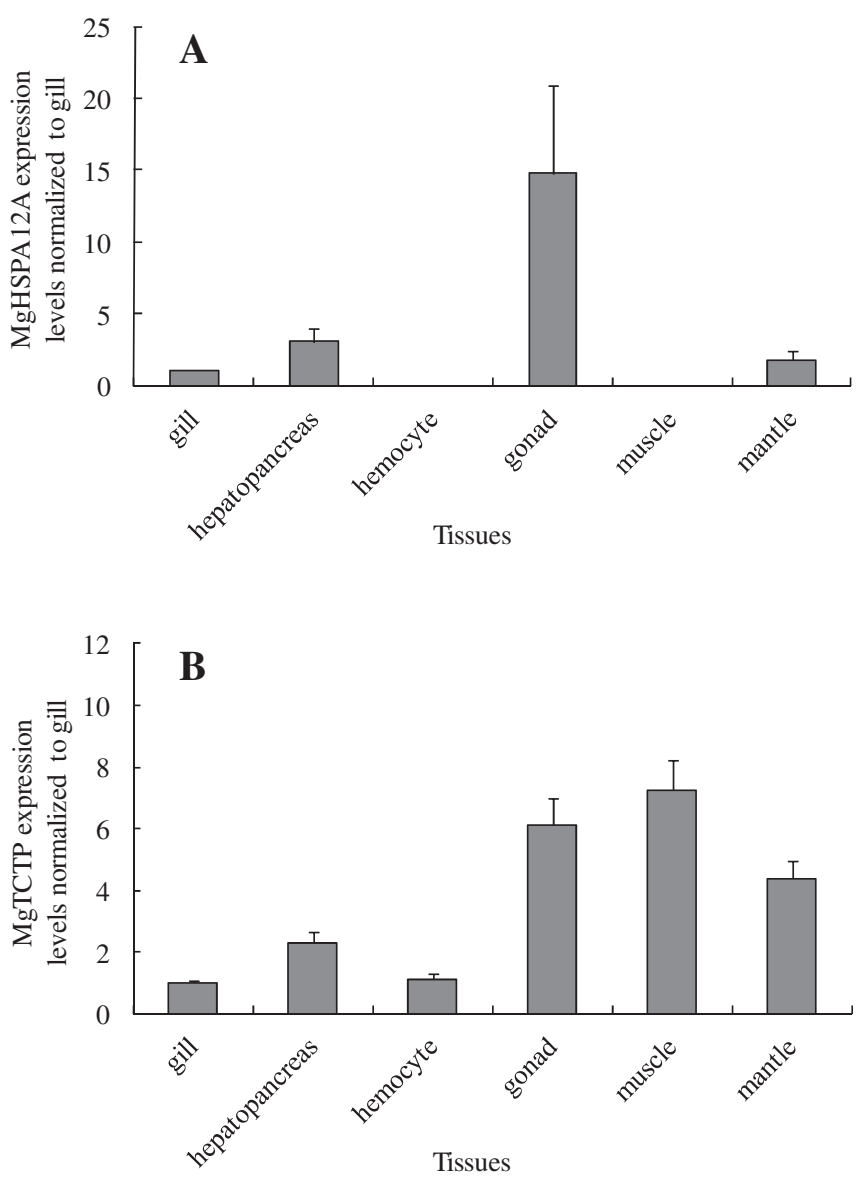

Fig. 3. Tissue distribution of the $\operatorname{MgHSPA} 12 \mathrm{~A}(\mathrm{~A})$ and $\operatorname{MgTCTP}(\mathrm{B})$ transcript measured by SYBR Green RT-PCR. The transcript levels in hemocytes, hepatopancreas, gonad, muscle, and mantle were normalized to that in gills. Each symbol and vertical bars represented the means $\pm \operatorname{SE}(n=6)$.

\subsection{Tissues distribution of MgHSPA12A and MgTCTP}

The MgHSPA12A transcript was mainly detected in the tissues of gonad, hepatopancreas, mantle, and gills (Fig. 3A), while no obvious expression was observed in hemocytes and muscle. As concerned to MgTCTP, the transcript was detected in all the examined tissues, including hepatopancreas, hemocytes, gills, gonad, muscle, and mantle. It was dominantly expressed in the tissue of muscle, and moderately expressed in gonad and mantle (Fig. 3B).

\subsection{The temporal profiles of MgHSPA12A and MgTCTP mRNA after $V$. anguillarum challenge and $C d$ exposure}

The expression profiles of MgHSPA12A and MgTCTP genes in hepatopancreas post $V$. anguillarum infection was shown in Fig. 4. Significant increase of MgTCTP expression level (1.34-fold compared with the control, $p<0.05$ ) was detected at $48 \mathrm{~h}$ post infection and no significant difference was observed between the control group and challenged groups for MgHSPA12A during the challenge period.

The temporal expression of MgHSPA12A and MgTCTP transcripts in hepatopancreas after cadmium exposure was shown in Fig. 5. Significant increase of MgHSPA12A expression (2.0-fold compared to the control, $p<0.01$ ) was observed at $48 \mathrm{~h}$ in $5 \mu \mathrm{g} / \mathrm{L}$ Cd-treated group. However, no significant difference of MgHSPA12A expression was observed at other time points compared with the blank group in both 5 and $50 \mu \mathrm{g} / \mathrm{L} \mathrm{Cd}$ exposed groups $(p<0.05)$. Concerning to MgTCTP transcript, the expression level decreased after exposed for $24 \mathrm{~h}(0.6$-fold, $p<0.05)$ and $96 \mathrm{~h}$ $(0.5$-fold, $p<0.05)$ compared with that of control group at both $\mathrm{Cd}$ concentrations.

\section{Discussion}

In this study, the cDNAs encoding MgHSPA12A and MgTCTP were identified from $M$. galloprovincialis, and these two proteins were confirmed by the sequence alignments, and phylogenetic analysis. As two of three HSP70 family signatures, IDLGTTFS (20aa27aa) and IVDLGGGTADITVV (226aa-239aa), were found in the deduced MgHSPA12A amino acid sequences, it strengthened the possibility of a functional specialization of HSPA12A within the group of HSP70 proteins [16]. This notion has been previously proposed by Han et al., who have reported the uniqueness of $\mathrm{C}$ terminal substrate binding domain in HSPA12A of mice [5]. For MgTCTP, existence of the characteristic motif IGANASAEEAA (47aa57aa) identified the homology of MgTCTP in the TCTP family [7]. Moreover, the typical sequence of an N-myristoylation site, GANASA (48aa-53aa), in MgTCTP sequence suggested the potential anti-apoptotic activity [30].

The ubiquities of HSPA12A and TCTP transcripts in different tissues have been reported in some other organisms, like mammals of human and mice for HSPA12A and mollusk of Venerupis
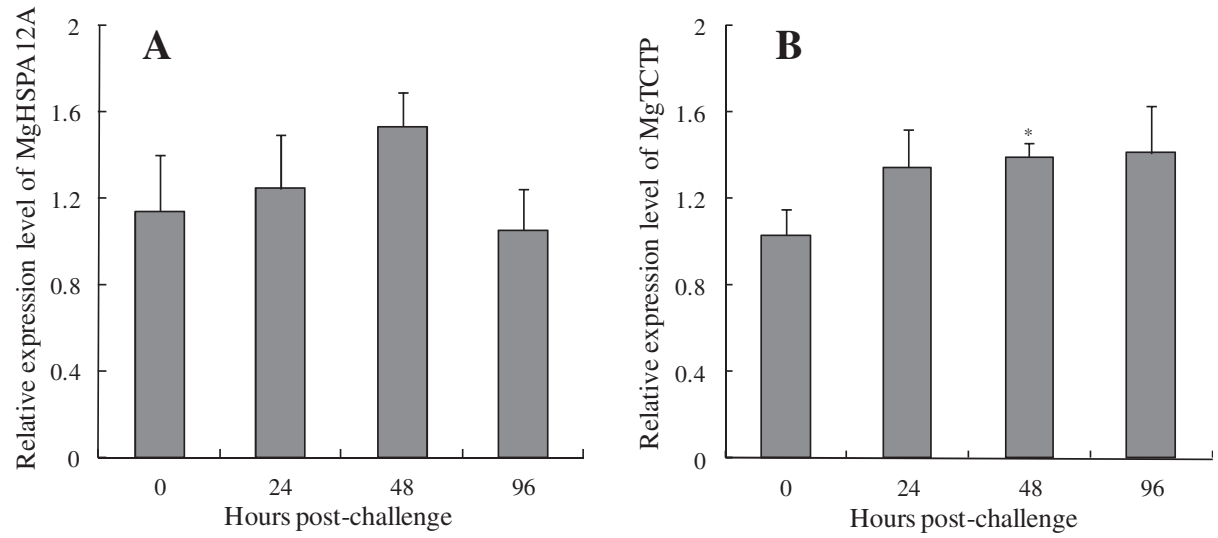

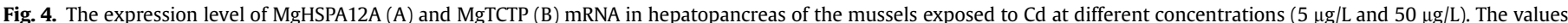

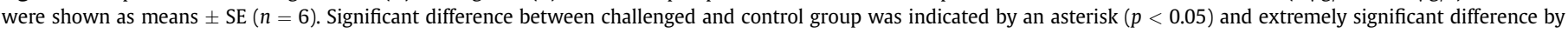
double asterisk $(p<0.01)$. 

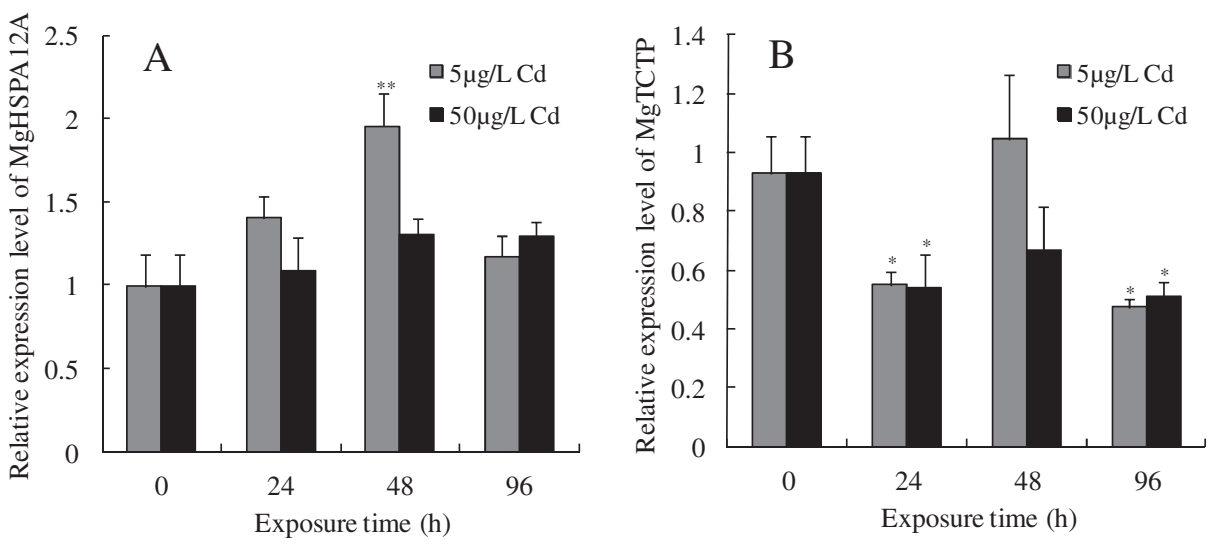

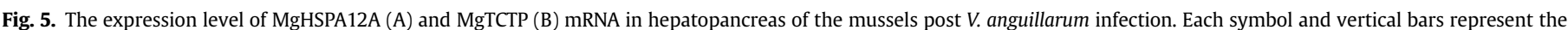

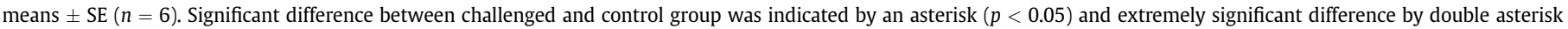
$(p<0.01)$.

philippinarum for TCTP $[5,6,11]$. In the present study, the expression of MgTCTP mRNA was dominantly detected in muscle, which was different from previous report on Venerupis philippinarum TCTP [11]. It was suggested that MgTCTP perhaps played an important role in muscle maintenance of $M$. galloprovincialis, as this tissue was frequently affected by heat, oxidative and mechanical stress [31]. Additionally, the high expression level of MgHSPA12A and MgTCTP in gonad perhaps indicated an essential role in reproductive processes, which was in agreement with Kappé et al., who found that some sHSPs were specially expressed in human testis [32].

In the aquatic environment, mollusks are frequently faced with pathophysiological and environmental stressors that can adversely affect their internal regulatory systems. It has been demonstrated that mollusk hepatopancreas represented a significant target for bacterial stimuli and toxic metal exposure [33-35]. HSP70 and TCTP, as members of HSPs family, were both implicated in the protection of cells against various abiotic stress and in the innate immune responses against bacterial challenge $[3,36]$. In the present study, no significant difference in the expression of MgHSPA12A was detected in hepatopancreas of mussels post $V$. anguillarum injection. This result was contrast with Cellura et al., who reported that HSP70 gene expression in M. galloprovincialis hemocytes was triggered by $V$. anguillarum challenge [13]. The difference perhaps further supported the notion that HSPA12 belongs to the HSP70 family but is distinct from the HSP70 family [5]. For MgTCTP, stimulated-expression in hepatopancreas was observed at $48 \mathrm{~h}$ post bacterial challenge. Similar phenomena were also observed in TCTP of $R$. philippinarum and SHSP22 in scallop challenged by $V$. anguillarum $[11,36]$. It was speculated that $V$. anguillarum was perhaps rapidly eliminated by the hemocytes [13], thus the amount of bacteria reached the hepatopancreas through the mussel circulatory system was not enough to produce significant effects. Moreover, the result might indicate that MgTCTP was more sensitive to $V$. anguillarum challenge than MgHSPA12A in hepatopancreas of M. galloprovincialis.

Concerning to $\mathrm{Cd}$ exposures, different stress responses were detected between these two genes, which indicated that they were involved in the detoxified system of mussels in different manners. The up-regulation of MgHSPA12A transcript at $48 \mathrm{~h}$ in $5 \mu \mathrm{g} / \mathrm{L} \mathrm{Cd}-$ treated group was perhaps related to the increased production of ROS, which was in agreement with previous observations in other organisms. For example, the expression of HSP70 and HSP90 in oysters exposed to Cd showed a significant enhancement [14,37]. However, no significant difference of MgHSPA12A expression was observed when $\mathrm{Cd}$ concentration increased to $50 \mu \mathrm{g} / \mathrm{L}$. It was deduced that strong oxidative stress caused by excess accumulation of Cd beyond a certain level in M. galloprovincialis drastically lowered the metabolic capacity, and then resulted in a less significant variation of MgHSPA12A expression profile in the $50 \mu \mathrm{g} / \mathrm{L} \mathrm{Cd}-$ treated samples than those of by $5 \mu \mathrm{g} / \mathrm{L}$ Cd-treated samples. Similar result was also reported on V. philippinarum treated with 10 , 20, and $40 \mu \mathrm{g} / \mathrm{L} \mathrm{Cu}$ and $\mathrm{Cd}$ respectively [25]. All these might be explained by the hormesis phenomenon in toxicology characterized by low-dose stimulatory and high-dose inhibitory responses $[39,40]$. As concerned to TCTP, there were few reports on TCTP expression profile of shellfish exposed to toxic metals. Recently, TCTP was reported to be an anti-apoptotic protein [38]. Hence the decrease of MgTCTP mRNA was perhaps consistent with their roles as negative regulators in apoptosis.

\section{Acknowledgements}

This research was supported by grant from National Natural Science Foundation of China (31172388) the 100 Talents Program of the Chinese Academy of Sciences, and Key Deployment Program of Chinese Academy of Sciences (KZZD-EW-14-03).

\section{Appendix A. Supplementary data}

Supplementary data related to this article can be found at http:// dx.doi.org/10.1016/j.fsi.2013.04.021.

\section{References}

[1] Beere HM. The stress of dying: the role of heat shock proteins in the regulation of apoptosis. Journal of Cell Science 2004;117:2641.

[2] Trent JD. A review of acquired thermotolerance, heat-shock proteins, and molecular chaperones in archaea. FEMS Microbiology Reviews 1996;18: 249-58.

[3] Bommer UA, Thiele BJ. The translationally controlled tumour protein (TCTP). The International Journal of Biochemistry \& Cell Biology 2004;36:379-85.

[4] Shu Y, Du Y, Wang J. Molecular characterization and expression patterns of Spodoptera litura heat shock protein 70/90, and their response to zinc stress. Comparative Biochemistry and Physiology - Part A: Molecular \& Integrative Physiology 2010.

[5] Han Z, Truong QA, Park S, Breslow JL. Two Hsp70 family members expressed in atherosclerotic lesions. Proceedings of the National Academy of Sciences 2003;100:1256.

[6] Pongrac JL, Middleton FA, Peng L, Lewis DA, Levitt P, Mirnics K. Heat shock protein 12A shows reduced expression in the prefrontal cortex of subjects with schizophrenia. Biological Psychiatry 2004;56:943-50.

[7] Thaw P, Baxter NJ, Hounslow AM, Price C, Waltho JP, Craven CJ. Structure of TCTP reveals unexpected relationship with guanine nucleotide-free chaperones. Nature Structural \& Molecular Biology 2001;8:701-4.

[8] Gnanasekar M, Dakshinamoorthy G, Ramaswamy K. Translationally controlled tumor protein is a novel heat shock protein with chaperone-like activity. Biochemical \& Biophysical Research Communications 2009;386:333-7. 
[9] Bommer UA, Borovjagin AV, Greagg MA, Jeffrey IW, Russell P, Laing KG, et al. The mRNA of the translationally controlled tumor protein P23/TCTP is a highly structured RNA, which activates the dsRNA-dependent protein kinase PKR. RNA 2002;8:478.

[10] Gachet Y, Tournier S, Lee M, Lazaris-Karatzas A, Poulton T, Bommer UA. The growth-related, translationally controlled protein P23 has properties of a tubulin binding protein and associates transiently with microtubules during the cell cycle. Journal of Cell Science 1999;112:1257.

[11] Li C, Oiu L, Ning X, Chen A, Oin S, Wu H, et al. The first molluscan TCTP in Venerupis philippinarum: molecular cloning and expression analysis. Fish \& Shellfish Immunology 2010;29:530-3.

[12] Margulis B, Guzhova I. Dual role of chaperones in the response of a cell and of a whole organism to stress. Tsitologiia 2009;51:219.

[13] Cellura C, Toubiana M, Parrinello N, Roch P. HSP70 gene expression in Mytilus galloprovincialis hemocytes is triggered by moderate heat shock and Vibrio anguillarum, but not by V. splendidus or Micrococcus lysodeikticus. Developmental \& Comparative Immunology 2006;30:984-97.

[14] Piano A, Valbonesi P, Fabbri E. Expression of cytoprotective proteins, heat shock protein 70 and metallothioneins, in tissues of Ostrea edulis exposed to heat and heavy metals. Cell Stress \& Chaperones 2004:134-42.

[15] Roccheri MC, Agnello M, Bonaventura R, Matranga V. Cadmium induces the expression of specific stress proteins in sea urchin embryos. Biochemical \& Biophysical Research Communications 2004;321:80-7.

[16] Song L, Wu L, Ni D, Chang Y, Xu W, Xing K. The cDNA cloning and mRNA expression of heat shock protein 70 gene in the haemocytes of bay scallop (Argopecten irradians, Lamarck 1819) responding to bacteria challenge and naphthalin stress. Fish \& Shellfish Immunology 2006;21:335-45.

[17] Araya MT, Markham F, Mateo DR, McKenna P, Johnson GR, Berthe FCJ, et al. Identification and expression of immune-related genes in hemocytes of softshell clams, Mya arenaria, challenged with Vibrio splendidus. Fish \& Shellfish Immunology 2010;29:557-64.

[18] Ripabelli G, Sammarco ML, Grasso GM, Fanelli I, Caprioli A, Luzzi I. Occurrence of Vibrio and other pathogenic bacteria in Mytilus galloprovincialis (mussels) harvested from Adriatic Sea, Italy. International Journal of Food Microbiology 1999;49:43-8.

[19] Yue X, Liu B, Xiang J, Jia J. Identification and characterization of the pathogenic effect of a Vibrio parahaemolyticus-related bacterium isolated from clam Meretrix meretrix with mass mortality. Journal of Invertebrate Pathology 2010;103:109-15.

[20] Parisi MG, Li H, Jouvet LBP, Dyrynda EA, Parrinello N, Cammarata M, et al. Differential involvement of mussel hemocyte sub-populations in the clearance of bacteria. Fish \& Shellfish Immunology 2008;25:834-40.

[21] Hervé-Fernández P, Houlbrèque F, Boisson F, Mulsow S, Teyssié JL, Oberhaënsli $\mathrm{F}$, et al. Cadmium bioaccumulation and retention kinetics in the Chilean blue mussel Mytilus chilensis: seawater and food exposure pathways. Aquatic Toxicology 2010;99:448-56.

[22] Kavun VY, Shulkin V, Khristoforova N. Metal accumulation in mussels of the Kuril Islands, north-west Pacific Ocean. Marine Environmental Research 2002;53:219-26.

[23] Szefer P, Kim BS, Kim CK, Kim EH, Lee CB. Distribution and coassociations of trace elements in soft tissue and byssus of Mytilus galloprovincialis relative to the surrounding seawater and suspended matter of the southern part of the Korean Peninsula. Environmental Pollution 2004;129:209-28.
[24] Zhang X. Investigation of pollution of $\mathrm{Pb}, \mathrm{Cd}, \mathrm{Hg}$, As in sea water and deposit of Bohai Sea area. Heilongjiang Environmental Journal 2001;25:87-90 [in Chinese with English abstract].

[25] Zhang L, Liu X, Chen L, You L, Pei D, Cong M, et al. Transcriptional regulation of selenium-dependent glutathione peroxidase from Venerupis philippinarum in response to pathogen and contaminants challenge. Fish \& Shellfish Immunology 2011.

[26] Wang Q, Zhang L, Zhao J, You L, Wu H. Two goose-type lysozymes in Mytilus galloprovincialis: possible function diversification and adaptive evolution. PLOS ONE 2012;7:9.

[27] Zorita I, Bilbao E, Schad A, Cancio I, Soto M, Cajaraville M. Tissue- and cellspecific expression of metallothionein genes in cadmium- and copperexposed mussels analyzed by in situ hybridization and RT-PCR. Toxicology and Applied Pharmacology 2007;220:186-96.

[28] Li C, Sun H, Chen A, Ning X, Wu H, Qin S, et al. Identification and characterization of an intracellular $\mathrm{Cu}, \mathrm{Zn}$-superoxide dismutase (icCu/Zn-SOD) gene from clam Venerupis philippinarum. Fish \& Shellfish Immunology 2010;28:499-503.

[29] Livak KJ, Schmittgen TD. Analysis of relative gene expression data using real-time quantitative PCR and the 2-[Delta][Delta] CT method. Methods 2001;25:402-8.

[30] Yang Y, Yang F, Xiong Z, Yan Y, Wang X, Nishino M, et al. An N-terminal region of translationally controlled tumor protein is required for its antiapoptotic activity. Oncogene 2005;24:4778-88.

[31] Sugiyama Y, Suzuki A, Kishikawa M, Akutsu R, Hirose T, Waye MMY, et al. Muscle develops a specific form of small heat shock protein complex composed of MKBP/HSPB2 and HSPB3 during myogenic differentiation. Journal of Biological Chemistry 2000;275:1095.

[32] Kappé G, Franck E, Verschuure P, Boelens WC, Leunissen JAM, De Jong WW. The human genome encodes $10 \alpha$-crystallin-related small heat shock proteins: HspB1-10. Cell Stress \& Chaperones 2003;8:53.

[33] Canesi L, Barmo C, Fabbri R, Ciacci C, Vergani L, Roch P, et al. Effects of Vibrio challenge on digestive gland biomarkers and antioxidant gene expression in Mytilus galloprovincialis. Comparative Biochemistry and Physiology Part C: Toxicology \& Pharmacology 2010;152:399-406.

[34] Company R, Serafim A, Bebianno M, Cosson R, Shillito B, Fiala-Médioni A. Effect of cadmium, copper and mercury on antioxidant enzyme activities and lipid peroxidation in the gills of the hydrothermal vent mussel Bathymodiolus azoricus. Marine Environmental Research 2004;58:377-81.

[35] Leonard SS, Harris GK, Shi X. Metal-induced oxidative stress and signal transduction. Free Radical Biology \& Medicine 2004;37:1921-42.

[36] Zhang L, Wang L, Zhao J, Qiu L, Song L, Dong C, et al. The responsive expression of heat shock protein 22 gene in zhikong scallop Chlamys farreri against bacterial challenge. Aquaculture Research 2010;41:257-66.

[37] Yong KC, Pil GJ, Cheol YC. Cadmium affects the expression of heat shock protein 90 and metallothionein mRNA in the Pacific oyster, Crassostrea gigas. Comparative Biochemistry and Physiology, Part C 2008;147:286-92.

[38] Gnanasekar M, Ramaswamy K. Translationally controlled tumor protein of Brugia malayi functions as an antioxidant protein. Parasitology Research 2007;101:1533-40.

[39] Zhang J, Wang X, Guo H, Wu J, Xue Y. Effects of water-soluble fractions of diesel oil on the antioxidant defenses of the goldfish, Carassius auratus. Ecotoxicology and Environmental Safety 2004;58:110-6.

[40] Calabrese EJ. Hormesis: a revolution in toxicology, risk assessment and medicine. EMBO Reports 2004;5:S37-40. 